\title{
Byproduct-induced in-situ formation of gold colloidal superparticles
}

\author{
Shaojue $\mathrm{Wu}^{1}$, Chung Yen $\mathrm{Ang}^{1}$, Zhong $\mathrm{Luo}^{1}$, Si Yu Tan ${ }^{1}$, Kim Truc Nguyen ${ }^{1}$ and Yanli Zhao ${ }^{1,2^{*}}$
}

\begin{abstract}
Current work presents a simple and efficient method for the preparation of gold superparticles. The self-assembly of gold nanoparticles into colloidal superparticles was realized in-situ during the preparation of nanoparticles when a type of thiol molecules were used as ligands. Further investigation reveals that $\boldsymbol{t}$-butylamine hydrochloride, an ionic byproduct generated during the redox reaction of precursors, plays a key role in the formation of gold superparticles. The polarity of monolayers made from these ligands on the nanoparticle surface allows the interaction of nanoparticles and the ionic byproduct, which is indispensable for the superparticle formation.
\end{abstract}

Superparticles (SPs) are colloidal assemblies of inorganic nanoparticles (NPs) [1-5]. Their unique structures endow them with interesting properties, which promise diverse applications such as controlled delivery and release $[6,7]$, magnetic separation $[8,9]$, dye sensitized solar cells [10], lithium ion batteries $[11,12]$, and noble metal catalysts [13]. To date, there are several methods to fabricate SPs in literature. In general, these methods can be sorted into two categories based on whether constituent NPs are isolated before the formation of SPs. In one category, SPs are fabricated via mediated assembly of purified NPs by employing driven forces like solvophobic interactions [14-21], hydrogen bonding [22], host-guest complexation $[6,7]$ and covalent bonding [23]. In the other category, SPs are formed in-situ in the synthetic solution of NPs. Notable examples are metal oxide SPs that were synthesized through high-temperature hydrolysis reactions [24-26]. A mechanism called surface tension inducing nanocrystallite aggregation was developed to describe the formation process [27]. Recently, a report revealed that SPs of different materials and complex structures could be synthesized in-situ as well, while the process was described with a different mechanism [28].

Nowadays, the formation pathways of SPs are still under exploration by researchers, since the understand- ing about them is not complete yet. Herein, we report a new phenomenon where monodisperse gold SPs (AuSPs, Scheme 1) were formed in-situ in the synthetic solution of gold NPs (AuNPs), when a special type of thiol ligands was used. These ligands feature a structure consisting of a tetraethylene glycol (TEG) chain with the two terminal hydroxyl groups replaced by a thiol group and a hydrocarbon segment respectively (HS-TEG-hydrocarbon, Scheme 1). Briefly, to a tetrahydrofuran (THF) solution containing the ligand and gold (III) chloride was injected a reducing agent $t$-butylamine borane (TBAB) that induced the formation of monodisperse AuNPs. Then, these AuNPs spontaneously aggregated to form AuSPs. Further investigation revealed that a byproduct, $t$-butylamine hydrochloride, generated from the redox reaction played a key role in the formation of AuSPs.

THF was used as a solvent, which can dissolve both $\mathrm{HAuCl}_{4}$ and $\mathrm{TBAB}$, so the reaction occurred in a singlephase system. HS-TEG-adamantane was the first ligand that we used. Upon the injection of TBAB/THF solution, monodisperse AuNPs (sample obtained after 30 s reaction, $8.0 \mathrm{~nm}$ in diameter, see Figs 1a and S1a in the Supplementary information (SI)) were formed quickly during the nucleation process, which is similar to other synthetic systems. However, the difference was that these AuNPs began to aggregate in solution in the following few minutes if no purification step was applied, as indicated by the UV-vis spectra (Fig. 1a). Transmission electron microscopy (TEM, Figs $1 \mathrm{~b}$ and c) and scanning electron microscopy (SEM, Fig. S2) images show that these AuNP aggregates (i.e., AuSPs) adopt a spherical morphology and are relatively uniform in size. Within these AuSPs, single AuNPs pack closely, the crystalline structure of which was confirmed by selected area electron diffraction (SAED, Fig. 1d). The formation of spherical assemblies is consistent with the emergence of the two absorption bands located at 549 and $630 \mathrm{~nm}$ in UV-vis

\footnotetext{
${ }^{1}$ Division of Chemistry and Biological Chemistry, School of Physical and Mathematical Sciences, Nanyang Technological University, Singapore 637371, Singapore

${ }^{2}$ School of Materials Science and Engineering, Nanyang Technological University, Singapore 639798, Singapore

"Corresponding author (email: zhaoyanli@ntu.edu.sg)
} 

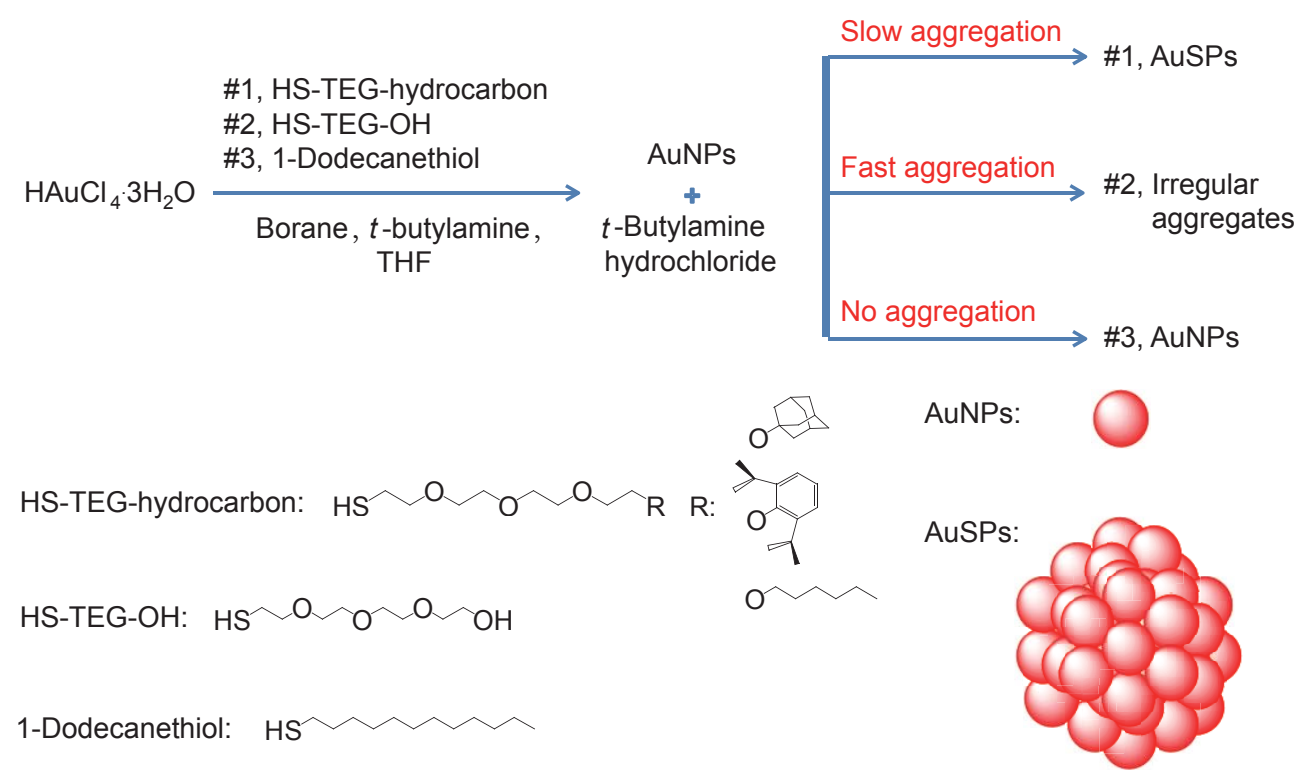

Scheme 1 Schematic illustration of the in-situ formation of AuSPs and the comparison of aggregation behavior of AuNPs when different thiol ligands were used.

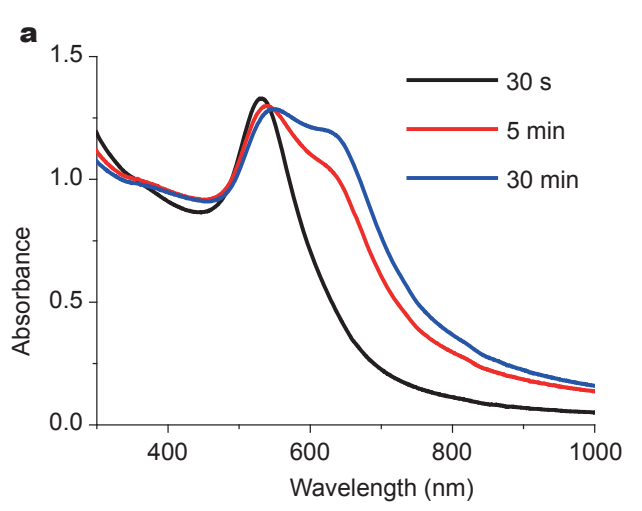

b
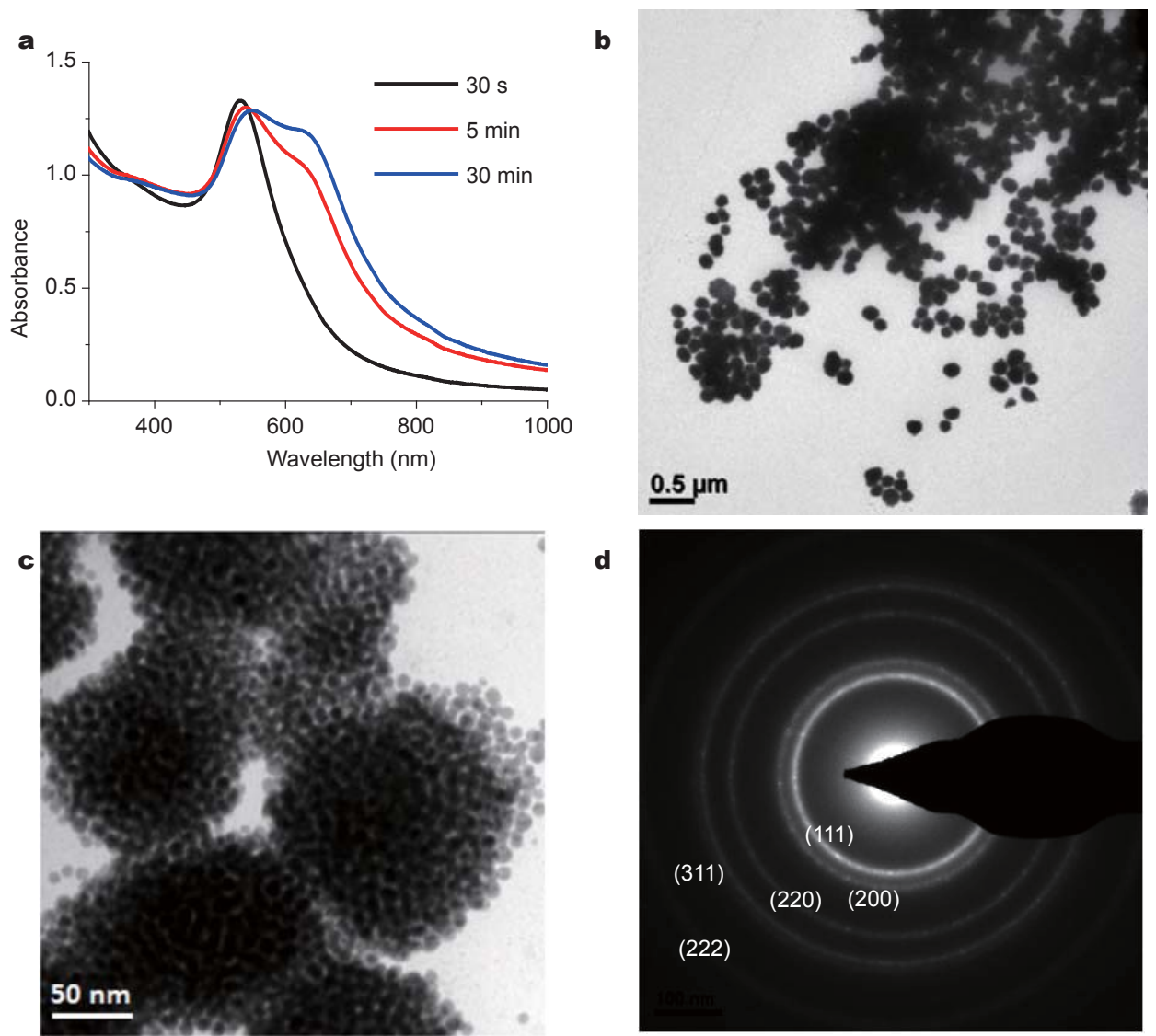

d

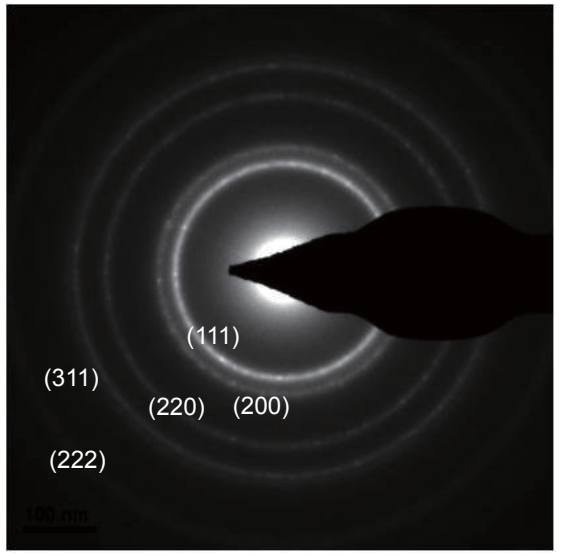

Figure 1 (a) UV-vis spectra for temporal evolution of the AuNP solution starting from the injection of TBAB solution at $30 \mathrm{~s}, 5 \mathrm{~min}$, and 30 min, (b and c) TEM images of AuSPs obtained after 30 min reaction, and (d) the corresponding SAED pattern of AuSPs. Ligand: HS-TEG-adamantane. 
spectrum of the 30 min sample. These AuSPs could further grow from $137 \pm 15$ to $159 \pm 19 \mathrm{~nm}$ from 5 to $30 \mathrm{~min}$, and maintained the monodispersity (size standard deviation $11 \%$ and $12 \%$ respectively, Fig. S3). Given that the size of AuNPs is about $8 \mathrm{~nm}$, this small size growth (159-137 = 22 $\mathrm{nm}$ ) of AuSPs equates to only one to two layers of AuNPs, implying that the finished state for the formation of AuSPs is nearly at the point of $5 \mathrm{~min}$. The TEM images of AuSPs with smaller sizes could not be obtained, because the aggregation of AuNPs was a fast process in which AuSPs with sizes above $100 \mathrm{~nm}$ could be reached in a very short time. Importantly, these AuSPs could be disassembled to free single AuNPs (8.8 nm in diameter, Fig. S1b) after the addition of 1-dodecanethiol, indicating that the constituent AuNPs were connected with neighboring ones by the ligand layer, and they were loosely held together by weak forces, rather than being crystallographically coalesced.

In addition to HS-TEG-adamantane, we also tested ligands with similar molecular structures to prove that this phenomenon was not exclusive to HS-TEG-adamantane. Firstly, we used 2,6-diisopropylbezene in place of adamantane as the terminal segment to afford HS-TEG-2,6-diisopropylbezene, since both 2,6-diisopropylbezene and adamantane are bulky groups. As expected, we observed the formation of AuSPs (Fig. 2a). In order to demonstrate that the formation of AuSPs is also not exclusive to the ligands with bulky terminal groups, we further employed one of the simplest hydrocarbon units, hexane, to replace adamantine for achieving HS-TEG-hexane. Again, we observed the formation of AuSPs (Figs $2 b$ and c). Based on these results, it was supposed that the formation of AuSPs could be generalized to some other ligands with the molecular structure of HS-TEG-hydrocarbon.

Since these ligands consist of two segments, i.e., TEG and hydrocarbon units, we then investigated the roles of these two segments in the formation of AuSPs. When we removed the TEG segment, for example using 1-dodecanethiol as the ligand, stable AuNP solution was obtained (Fig. S4). Thus, the middle TEG segment is needed for the aggregation. When we removed the hydrocarbon segment, for example using HS-TEG-OH as the ligand, the formed AuNPs aggregated immediately and completely upon the injection of TBAB to become precipitates, leaving a colorless supernatant. These AuNP precipitates were large pieces of random aggregates with no regular morphology (Fig. S5a). Thus, the terminal hydrocarbon segment is indispensable for AuSPs to be monodisperse and regular in morphology. Since hydroxyl group is usually associated with hydrogen bonding interaction, the fast aggregation may result from strong hydrogen bonding between AuNPs in THF. When THF was replaced with water, a solvent with stronger hydrogen bonding ability than THF, these aggregates were disassembled completely to form free single AuNPs (Fig. S5b). In fact, changing the hydroxyl group to the shortest methyl group (i.e., HS-TEG-methane) significantly reduced the rate of aggregation in THF, while the obtained aggregates were slightly more regular (Fig. S6). Therefore, the role of a hydrocarbon terminal group should lie in the elimination of strong hydrogen bonding interaction between AuNPs in THF, which may enable AuNPs to rearrange themselves to energetically more favorable positions, thus leading the aggregates to adopt the energetically more favorable spherical morphology $[18,19]$. Since the surfaces of AuNPs are passivated by the thiol groups, there is no $\mathrm{SH}$ group available for hydrogen bonding interactions.

Then, we need to explain why the aggregation occurred in the synthetic solution of AuNPs when these HS-TEG-hydrocarbon ligands were used. One possible explanation is that the ligand layers are not compatible with the solvent. THF is reported to be not a good solvent for polyethylene glycols while a good solvent for hydrocarbons [29,30].
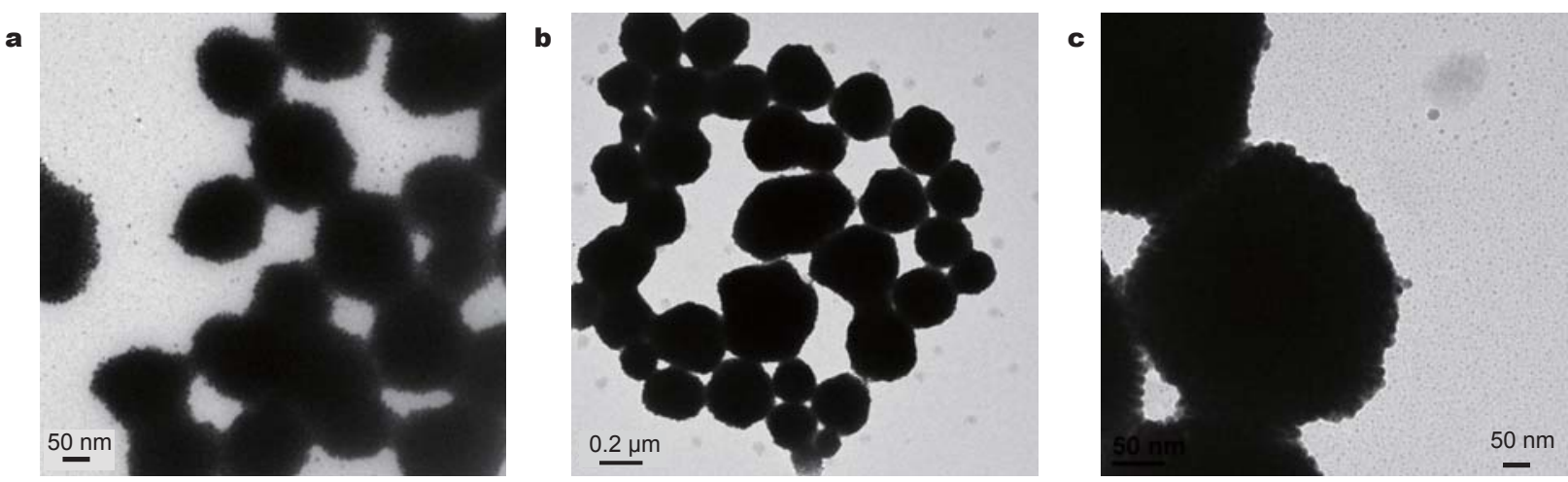

Figure 2 TEM images of AuSPs with (a) HS-TEG-2,6-diisopropylbezene as a ligand, and (b and c) HS-TEG-hexane as a ligand. 
However, this explanation was excluded by the observation that AuNPs coated with HS-TEG-adamantane (denoted as AuNPs@HS-TEG-adamantane) could in fact be stably dispersed in THF after being purified from the synthetic solution. This observation is reasonable, as the solubility of AuNPs is largely determined by the chemical structure of the terminal groups of ligands. The inherent solubility of AuNPs@HS-TEG-adamantane in THF suggests that chemical species remained in the synthetic solution may play a role in the AuNP aggregation. Experimentally, we found that, accompanied with AuNP aggregation, some precipitates were separated out from the synthetic solution. Based on ${ }^{1} \mathrm{H}$ NMR (Fig. S7) and X-ray photoelectron spectroscopy (XPS, Fig. S8) analyses, we identified these precipitates as $t$-butylamine hydrochloride, the ionicity of which explained why they were separated out from THF, a moderately polar solvent. Thus, the chemical species remained in the solution phase were identified as mainly unreacted HS-TEG-adamantane and $t$-butylamine (Fig. S9). $t$-Butylamine hydrochloride is a byproduct of the redox reaction between $\mathrm{HAuCl}_{4}$ and TBAB. During the reaction, $\mathrm{HCl}$ liberated from $\mathrm{HAuCl}_{4}$ and $t$-butylamine liberated from TBAB could undergo acid-base reaction to form $t$-butylamine hydrochloride.

Based on the composition analysis, we believe that $t$-butylamine is a necessary component for the AuNP aggregation, as it exists in both the solution and precipitate phases. To prove it, we carried out a control experiment using $\mathrm{BH}_{3}$ in place of TBAB as the reducing agent. AuNPs were synthesized without any aggregation at any time points in this case (Fig. 3a). However, when certain amount of $t$-butylamine was added to the above solution, these AuNPs aggregated immediately. At the same time, we also observed the generation of some precipitates, a phenomenon which was similar to the original reaction using TBAB. TEM images of the formed aggregates show that the spherical subdomains consisting of AuNPs pack together to form large aggregates (Figs $3 \mathrm{~b}$ and S10). These spherical subdomains have a striking resemblance to AuSPs (Figs 1 and 2). Thus, this control experiment identified $t$-butylamine as the key component in the formation of AuSPs. It should be noted that when triethylamine was added in place of $t$-butylamine, a similar phenomenon was observed, indicating that it is the amino group, instead of the $t$-butyl structure, which plays the key role.

Next, we need to understand how $t$-butylamine works to induce the AuNP aggregation. Since the added quantity of thiol ligands exceeds that of either $t$-butylamine (calculated based on the quantity of TBAB) or $\mathrm{HAuCl}_{4}$ in the synthetic solution, and the affinity of thiols toward AuNPs is much stronger than that of amines, direct bonding of $t$-butylamine on AuNP surface is not reasonable. We then performed a control experiment. To a THF solution of AuNPs@HS-TEG-adamantane (synthesized with $\mathrm{BH}_{3}$ as reducing agent and then purified for use, see SI for more details), $200 \mu \mathrm{L}$ of $t$-butylamine was added. No noticeable aggregation occurred at this step, proving that the aggregation of AuNPs was not resulted from direct bonding of $t$-butylamine and AuNPs. However, when dry $\mathrm{HCl}$ gas was bubbled into the solution, there was a significant drop of AuNP concentration (Fig. 4) and simultaneous generation of some precipitates (a mixture of AuNP aggregates and $t$-butylamine hydrochloride). Without the addition of $t$-butylamine, bubbling $\mathrm{HCl}$ gas to the AuNP solution caused no obvious change. Although these AuNP aggregates were random ones without any regular morphology in this case, their formation did suggest that reacting with $\mathrm{HCl}$ to form $t$-butylamine hydrochloride was the way that
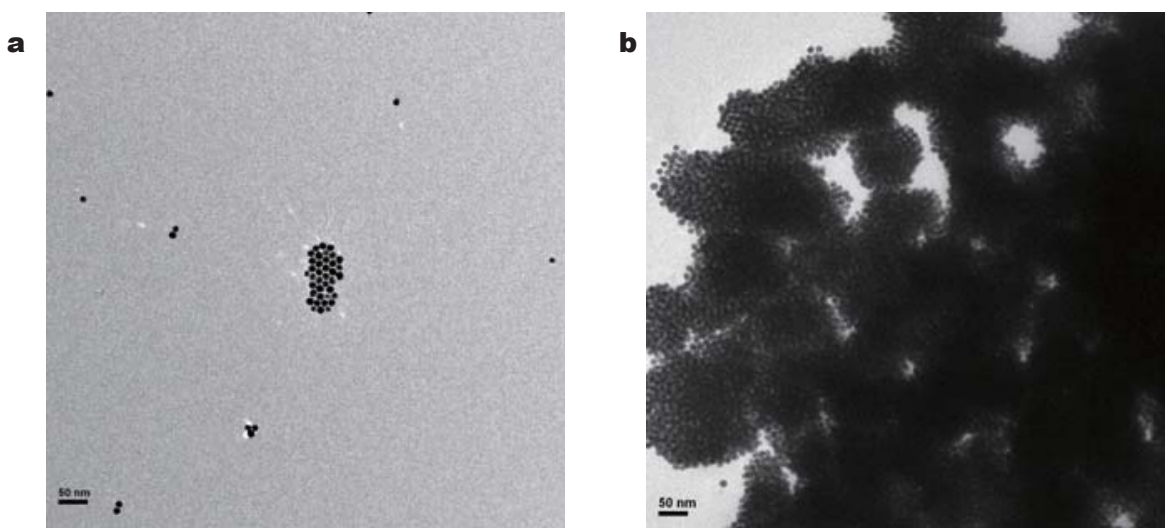

Figure 3 TEM images of (a) free single AuNPs obtained by using $\mathrm{BH}_{3}$ in place of TBAB as a reducing agent, and (b) AuNP aggregates obtained after adding $t$-butylamine into the AuNP solution. 


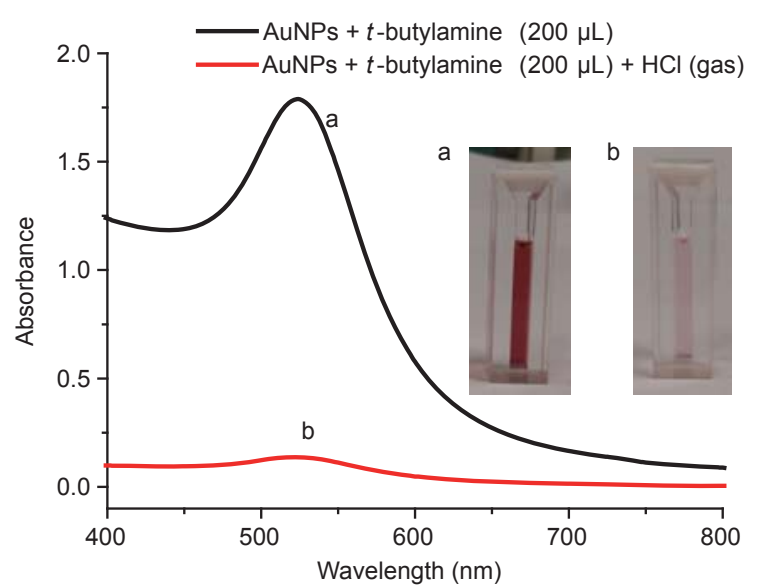

Figure 4 UV-vis spectra of AuNP/THF solution with only $t$-butylamine added (curve a) and then $\mathrm{HCl}$ gas introduced (curve b). Inset: photographs of the corresponding AuNP solution before (cuvette a) and after (cuvette b) introducing $\mathrm{HCl}$ gas.

t-butylamine could induce the AuNP aggregation and thus AuSP formation.

From the above discussions, we could identify two key ingredients for the formation of AuSPs, i.e., the chemical structure of the ligands (HS-TEG-hydrocarbon) and generation of the byproduct ( $t$-butylamine hydrochloride). Thus, AuSP formation should be a result of the interaction between the ligand monolayers of AuNPs and the in-situ generated $t$-butylamine hydrochloride, denoted as NPbyproduct interaction. As above-mentioned, AuNPs did not aggregate when 1-dodecanethiol was used as a ligand, meaning that there was no NP-byproduct interaction in this case. This is understandable, since 1-dodecanethiol monolayer has a low polarity. For comparison, $t$-butylamine hydrochloride is a salt with relatively high ionicity. The large difference in polarity between them explains the absence of NP-byproduct interaction. On the other hand, HS-TEG-hydrocarbon molecules have a TEG segment possessing a certain degree of polarity, allowing for the monolayers to interact with $t$-butylamine hydrochloride. The NP-byproduct interaction in this case is proposed, deriving from the polarity of the HS-TEG-hydrocarbon monolayer and the ionicity of $t$-butylamine hydrochloride. This interaction could make some $t$-butylamine hydrochloride molecules (or their small aggregates) adsorbed onto the surface of AuNPs, rendering AuNPs solvophobic in THF and ultimately forming AuSPs through the aggregation.

In summary, we have developed a methodology for in-situ formation of AuSPs. The generation of AuSPs requires the use of a certain type of ligands with the structure of HS-TEG-hydrocarbon, THF as solvent, and the genera- tion of ionic byproduct $t$-butylamine hydrochloride. The polarity of the HS-TEG-hydrocarbon monolayers plays a key factor for the occurrence of NP-byproduct interaction, which induces the aggregation of NPs and formation of SPs. The current work provides an alternative choice when similar systems are used in the preparation of SPs. As SPs are an important member of functional nanomaterials, research on their formation pathways is of great importance, and the present work may lead to a better understanding of the SP formation. As these AuSPs have a size range between 100 and $200 \mathrm{~nm}$ and possess largely enhanced absorption above $600 \mathrm{~nm}$, they are potentially suitable for the applications in photothermal treatment of cancer cells [31].

Received 10 September 2015; accepted 8 October 2015; published online 6 November 2015

1 Wang T, LaMontagne D, Lynch J, Zhuang J, Cao YC. Colloidal superparticles from nanoparticle assembly. Chem Soc Rev, 2013, 42: 2804-2823

2 Xia Y, Tang Z. Monodisperse inorganic supraparticles: formation mechanism, properties and applications. Chem Commun, 2012, 48: 6320-6336

3 Zhuang J, Wu H, Yang Y, Cao YC. Controlling colloidal superparticle growth through solvophobic interactions. Angew Chem Int Ed, 2008, 47: 2208-2212

4 Wang PP, Yu Q, Long Y, et al. Multivalent assembly of ultrasmall nanoparticles: one-, two-, and three-dimensional architectures of 2 nm gold nanoparticles. Nano Res, 2012, 5: 283-291

5 Goubet N, Pileni MP. Negative supracrystals inducing a FCC-BCC transition in gold nanocrystal superlattices. Nano Res, 2014, 7: 171-179

6 Wang S, Chen KJ, Wu TH, et al. Photothermal effects of supramolecularly assembled gold nanoparticles for the targeted treatment of cancer cells. Angew Chem Int Ed, 2010, 49: 3777-3781

7 Lee JH, Chen KJ, Noh SH, et al. On-demand drug release system for in-vivo cancer treatment through self-assembled magnetic nanoparticles. Angew Chem Int Ed, 2013, 52: 4384-4388

8 Ge J, Hu Y, Biasini M, Beyermann WP, Yin Y. Superparamagnetic magnetite colloidal nanocrystal clusters. Angew Chem Int Ed, 2007, 46: 4342-4345

9 Deng Y, Qi D, Deng C, Zhang X, Zhao D. Superparamagnetic high-magnetization microspheres with an $\mathrm{Fe}_{3} \mathrm{O}_{4} @ \mathrm{SiO}_{2}$ core and perpendicularly aligned mesoporous $\mathrm{SiO}_{2}$ shell for removal of microcystins. J Am Chem Soc, 2007, 130: 28-29

10 Chou TP, Zhang Q, Fryxell GE, Cao GZ. Hierarchically structured $\mathrm{ZnO}$ film for dye-sensitized solar cells with enhanced energy conversion efficiency. Adv Mater, 2007, 19: 2588-2592

11 Lee $\mathrm{SH}$, Yu SH, Lee JE, et al. Self-assembled $\mathrm{Fe}_{3} \mathrm{O}_{4}$ nanoparticle clusters as high-performance anodes for lithium ion batteries via geometric confinement. Nano Lett, 2013, 13: 4249-4256

12 Liu N, Lu Z, Zhao J, et al. A pomegranate-inspired nanoscale design for large-volume-change lithium battery anodes. Nat Nanotechnol, 2014, 9: 187-192

13 Chen C, Nan C, Wang D, et al. Mesoporous multicomponent nanocomposite colloidal spheres: ideal high-temperature stable model catalysts. Angew Chem Int Ed, 2011, 50: 3725-3729

$14 \mathrm{Xu} \mathrm{H}$, Cui L, Tong N, Gu H. Magnetic fluorescent delivery vehicle using uniform mesoporous silica spheres embedded with mono- 
disperse magnetic and semiconductor nanocrystals. J Am Chem Soc, 2006, 128: 15582-15583

15 Bai F, Wang D, Huo Z, et al. A versatile bottom-up assembly approach to colloidal spheres from nanocrystals. Angew Chem Int Ed, 2007, 46: 6650-6653

16 Zhuang J, Wu H, Yang Y, Cao YC. Supercrystalline colloidal particles from artificial atoms. J Am Chem Soc, 2007, 129: 14166-14167

17 Zhuang J, Shaller AD, Lynch J, et al. Cylindrical superparticles from semiconductor nanorods. J Am Chem Soc, 2009, 131: 6084-6085

18 Wang T, Wang X, LaMontagne D, et al. Shape-controlled synthesis of colloidal superparticles from nanocubes. J Am Chem Soc, 2012, 134: $18225-18228$

19 Wang T, Zhuang J, Lynch J, et al. Self-assembled colloidal superparticles from nanorods. Science, 2012, 338: 358-363

20 Lacava J, Born P, Kraus T. Nanoparticle clusters with lennard-jones geometries. Nano Lett, 2012, 12: 3279-3282

21 Sánchez-Iglesias A, Grzelczak M, Altantzis T, et al. Hydrophobic interactions modulate self-assembly of nanoparticles. ACS Nano, 2012, 6: 11059-11065

22 Boal AK, Ilhan F, DeRouchey JE, et al. Self-assembly of nanoparticles into structured spherical and network aggregates. Nature, 2000, 404: 746-748

23 Maye MM, Luo J, Lim IIS, et al. Size-controlled assembly of gold nanoparticles induced by a tridentate thioether ligand. J Am Chem Soc, 2003, 125: 9906-9907

24 Hu X, Gong J, Zhang L, Yu JC. Continuous size tuning of monodisperse $\mathrm{ZnO}$ colloidal nanocrystal clusters by a microwave-polyol process and their application for humidity sensing. Adv Mater, 2008, 20: 4845-4850

25 Narayanaswamy A, Xu H, Pradhan N, Peng X. Crystalline nanoflowers with different chemical compositions and physical properties grown by limited ligand protection. Angew Chem Int Ed, 2006, 45: 5361-5364

26 Matijevic E. Monodispersed metal (hydrous) oxides-a fascinating field of colloid science. Acc Chem Res, 1981, 14: 22-29
27 Privman V, Goia DV, Park J, Matijević E. Mechanism of formation of monodispersed colloids by aggregation of nanosize precursors. J Colloid Interface Sci, 1999, 213: 36-45

28 Xia Y, Nguyen TD, Yang M, et al. Self-assembly of self-limiting monodisperse supraparticles from polydisperse nanoparticles. Nat Nanotechnol, 2011, 6: 580-587

29 Gitsov I, Fréchet JMJ. Stimuli-responsive hybrid macromolecules: novel amphiphilic star copolymers with dendritic groups at the periphery. J Am Chem Soc, 1996, 118: 3785-3786

30 Niikura K, Iyo N, Higuchi T, et al. Gold nanoparticles coated with semi-fluorinated oligo (ethylene glycol) produce sub-100 nm nanoparticle vesicles without templates. J Am Chem Soc, 2012, 134: 7632-7635

31 Niikura K, Iyo N, Matsuo Y, Mitomov H, Ijiro K. Sub-100 nm gold nanoparticle vesicles as a drug delivery carrier enabling rapid drug release upon light irradiation. ACS Appl Mater Interfaces, 2013, 5: 3900-3907

Acknowledgements This work was supported by the National Research Foundation (NRF), Prime Minister's Office, Singapore under its Campus for Research Excellence and Technological Enterprise (CREATE) Programme-Singapore Peking University Research Centre for a Sustainable Low-Carbon Future, as well as the NTU-A*Star Silicon Technologies Centre of Excellence under the program grant No. 11235100003 .

Author contributions Wu S and Zhao Y conceived the study and wrote the manuscript. Ang CY, Luo Z, and Tan SY participated in the synthesis. Nguyen KT assisted to carry out the TEM studies. All authors contributed to the discussion.

Conflict of interest The authors declare that they have no conflict of interest.

Supplementary information Experimental details and supporting data are available in the online version of the paper. 


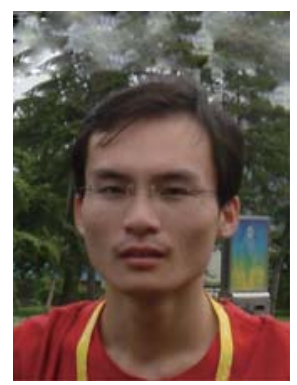

Shaojue Wu is currently a fourth-year PhD student under the supervision of Associate Professor Yanli Zhao at Nanyang Technological University, Singapore. His research focuses on the preparation and self-assembly of metal nanoparticles for sensing applications.

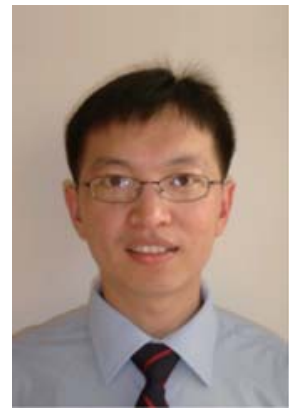

Yanli Zhao is currently an associate professor at Nanyang Technological University, Singapore. He received his BSc degree in chemistry and $\mathrm{PhD}$ degree in physical chemistry under the supervision of Prof. Yu Liu from Nankai University in 2000 and 2005, respectively. He was a postdoctoral scholar in Prof. Sir Fraser Stoddart's group from Oct. 2005 to Nov. 2008, and from Dec. 2008 to Dec. 2009 in Prof. Jeffrey Zink's group at the University of California Los Angeles, and subsequently at Northwestern University from Jan. 2010 to Aug. 2010. He has published over 190 peer-reviewed scientific papers, and received several awards including the TR35@Singapore Award in 2012 and Singapore National Research Foundation Fellowship in 2010. His current research focuses on the development of integrated systems for diagnostics and therapeutics, as well as porous materials for gas storage and catalysis.

中文摘要 本文提供了一个简单有效的方法制备超级金纳米粒子. 制备金纳米粒子的过程中加入一类颈基配体, 得到的金纳米粒子会 自聚集形成超级金纳米粒子. 进一步的研究发现,一个氧化还原反应的副产物叔丁胺盐酸盐在形成超级金纳米粒子的过程中起到了重 要的作用. 附着于金纳米粒子表面的配体与叔丁胺盐酸盐相互作用, 促进了超级金纳米粒子的形成. 